Med Klin Intensivmed Notfmed 2018 · 113:401-408 https://doi.org/10.1007/s00063-016-0182-8

Eingegangen: 12. Oktober 2015

Überarbeitet: 2. März 2016

Angenommen: 15. März 2016

Online publiziert: 5. Juli 2016

(c) Der/die Autor(en) 2016

Redaktion

G. Heinz, Wien

M. Buerke, Siegen

CrossMark

\title{
T. Staudinger
}

Universitätsklinik für Innere Medizin I, Intensivstation 13.i2, Allgemeines Krankenhaus der Stad

Wien/Medizinische Universität Wien, Wien, Österreich

\section{Extrakorporale Lungenersatz- verfahren - was gibt es Neues, was kommt?}

das - derzeit nicht mehr auf dem Markt befindliche - DeCAP-Smart ${ }^{\circledR}$-System (Hemodec, San Pietro Vimiano, Italien) mit Rollerpumpe und der arteriovenösen pumpenlosen ILA ${ }^{\circledR}$ (,interventional lung assist“, Novalung, Heilbronn, Deutschland), erfolgt der Antrieb mittels für die jeweiligen Blutflüsse optimierten magnetisch angetriebenen blutatraumatischen Zentrifugalpumpen mit minimiertem Füllungsvolumen. Das Material moderner Gasaustauschmembranen ist heute Polymethylpenten, das im Gegensatz zum bis vor einigen Jahren verwendeten Polypropylen plasmadicht bleibt und eine wesentlich längere Lebensdauer hat. Heparinbeschichtete Schlauchsysteme sind seit etwa einem Jahrzehnt Standard und verringern die Aktivierung von Gerinnungssystem sowie Entzündungskaskaden [23]. Vorgefertigte All-in-oneSysteme für das jeweilig gewünschte Einsatzspektrum werden für die meisten Geräte angeboten und ersparen die früher übliche „Bastelei“ mit dem Risiko einer bakteriellen Kontaminierung und von Blutverlusten. Die Zulassungsdauer solcher modernen Systeme liegt in der Regel zwischen 2 und 4 Wochen. Neben den klassischen ECMO-Konsolen hat in den letzten Jahren eine Reihe von $\mathrm{CO}_{2}$-Eliminationsverfahren den Weg auf den Markt gefunden bzw. steht vor der Einführung. Eine Auswahl solcher Verfahren mit ihren wesentlichen Charakteristiken findet sich in • Tab. 1.

\section{Erweiterung des Spektrums, differenzierte Konfiguration}

Um die unterschiedlichen Zielsetzungen und die dafür vorgesehene Konfiguration der Systeme unterscheiden zu können, muss man das Prinzip des Gasaustauschs über eine extrakorporale Membran verstanden haben: Die Oxygenierungsleistung hängt von der Menge des Bluts ab, das pro Zeit durch die Membran gepumpt wird, und die $\mathrm{CO}_{2}$-Elimination sowohl vom Blutfluss als auch vom Gasfluss (in der Regel Sauerstoff), der die Hohlfasern der Membranen durchströmt. Eine klinisch relevante $\mathrm{CO}_{2}$-Elimination beginnt bei Blutflüssen um $300 \mathrm{ml} / \mathrm{min}$ und erreicht ihr Maximum (d.h. Steady State zwischen Elimination und Neuproduktion) bei etwa $1500-2000 \mathrm{ml} / \mathrm{min}$. $\mathrm{Zu}$ sätzlich wird umso mehr $\mathrm{CO}_{2}$ entfernt, je mehr Gas durch die Membran strömt und damit das aus dem Blut in die Kapillare des Membranoxygenators diffundierte $\mathrm{CO}_{2}$ rascher abtransportiert, um somit den Konzentrationsgradienten zwischen Blut- und Gasphase maximal zu halten. $\mathrm{Ab} 1500-2000 \mathrm{ml} / \mathrm{min}$ ist die $\mathrm{CO}_{2}$-Elimination nur mehr vom Gasfluss abhängig. Das bedeutet, vereinfacht dargestellt, dass zur reinen $\mathrm{CO}_{2}$-Elimination Systeme ausreichen, die keine hohen Blutflüsse generieren müssen, während eine extrakorporale Oxygenierung Systeme mit der Möglichkeit, hohe Blutflüsse bis zu 6 l/min oder sogar mehr zu generieren, erfordert.

Die klassischen High-flow-Gasaustauschverfahren - venovenöse und venoarterielle ECMO - sind mittlerweile 

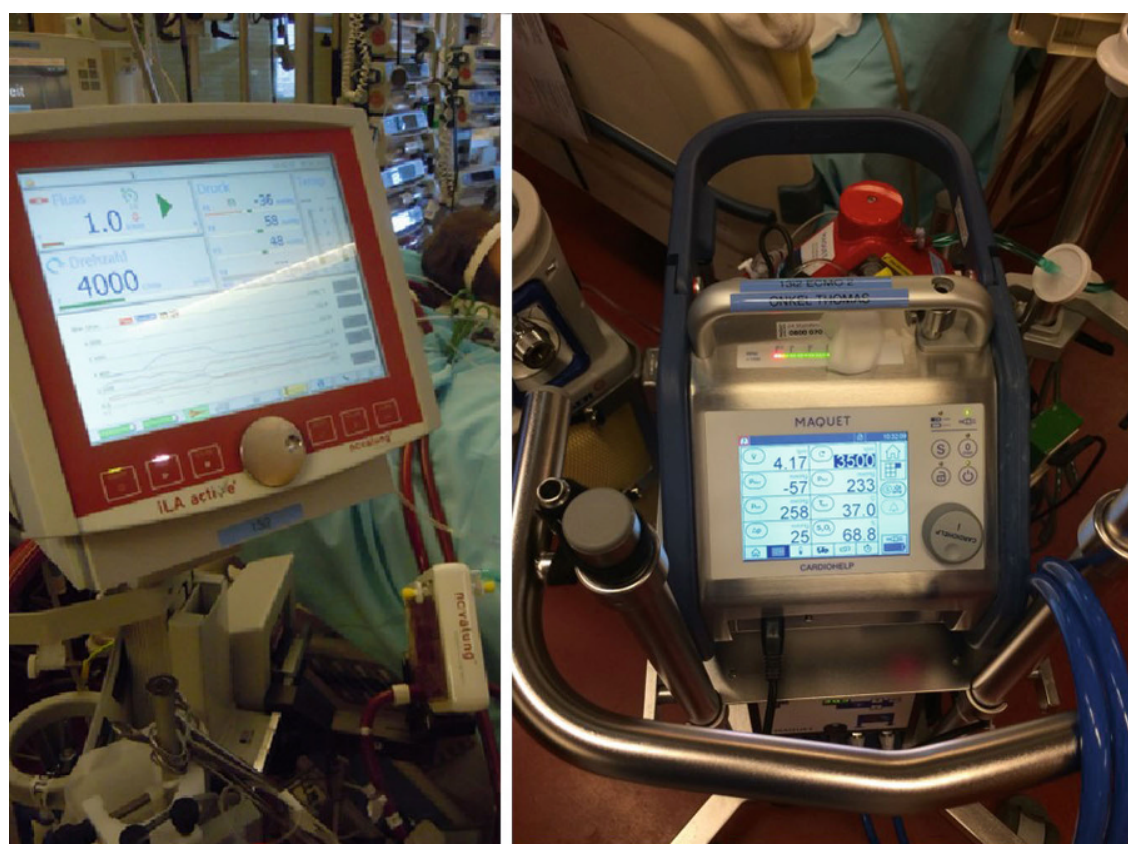

Abb. 1 A Beispiele moderner ECLS-Konsolen: links die iLA activve ${ }^{\circledR}$ (Novalung, Heilbronn, Deutschland), rechts die Cardiohelp ${ }^{\circledR}$ (Maquet, Rastatt, Deutschland) mit integriertem Blutfluss- und Druckmonitoring sowie der Möglichkeit von Interventionen durch die Gerätesoftware (z. B. Pumpenstopp bei Luftblasendetektion). Bei Cardiohelp ${ }^{\circledR}$ zusätzlich kontinuierlich $\mathrm{S}_{1} \mathrm{O}_{2}$, Bluttemperatur, Hämoglobin und Hämatokrit

\begin{tabular}{|c|c|c|c|c|}
\hline Name & Firma & Pumpe/Antrieb & Blutfluss & Monitoring \\
\hline ILA & $\begin{array}{l}\text { Novalung, Heil- } \\
\text { bronn, Deutsch- } \\
\text { land }\end{array}$ & $\begin{array}{l}\text { Pumpenlos (arterio- } \\
\text { venöse Druckdiffe- } \\
\text { renz) }\end{array}$ & $\begin{array}{l}\text { Je nach Kanülen } \\
\text { und Blutdruck } \\
600-1500 \mathrm{ml} / \mathrm{min}\end{array}$ & Blutfluss \\
\hline $\begin{array}{l}\text { iLA } \\
\text { activve }\end{array}$ & $\begin{array}{l}\text { Novalung, Heil- } \\
\text { bronn, Deutsch- } \\
\text { land }\end{array}$ & Zentrifugalpumpe & $\begin{array}{l}\text { Modulsystem; je } \\
\text { nach Kanülen- und } \\
\text { Membranwahl } \\
1-6 \mathrm{l} / \mathrm{min}\end{array}$ & $\begin{array}{l}\text { Blutfluss, } \\
\text { Drücke, Luft- } \\
\text { blasen }\end{array}$ \\
\hline $\begin{array}{l}\text { Cardiohelp- } \\
\text { PALP }\end{array}$ & $\begin{array}{l}\text { Maquet, Rastatt, } \\
\text { Deutschland }\end{array}$ & Zentrifugalpumpe & $\begin{array}{l}\text { Je nach Kanülen bis } \\
2,8 \mathrm{l} / \mathrm{min}\end{array}$ & $\begin{array}{l}\text { Blutfluss, Drücke, } \\
\text { Luftblasen, } \mathrm{S}_{\mathrm{v}} \mathrm{O}_{2}, \\
\mathrm{Hgb}, \text { Bluttempe- } \\
\text { ratur }\end{array}$ \\
\hline $\begin{array}{l}\text { DeCAP } \\
\text { Smart }\end{array}$ & $\begin{array}{l}\text { Hemodec, San } \\
\text { Pietro Vimiano, } \\
\text { Italien }\end{array}$ & Rollerpumpe & Bis $400 \mathrm{ml} / \mathrm{min}$ & Blutfluss, Drücke \\
\hline $\begin{array}{l}\text { Hemolung } \\
\text { RAS }\end{array}$ & $\begin{array}{l}\text { A-Lung Technolo- } \\
\text { gies, Pittsburgh, } \\
\text { USA }\end{array}$ & Zentrifugalpumpe & Bis $550 \mathrm{ml} / \mathrm{min}$ & $\begin{array}{l}\text { Blutfluss, } \\
\mathrm{CO}_{2} \text {-Elimination, } \\
\text { Luftblasen }\end{array}$ \\
\hline $\begin{array}{l}\text { Prismaflex- } \\
\mathrm{ECCO}_{2}-R\end{array}$ & $\begin{array}{l}\text { Gambro, Hechin- } \\
\text { gen, Deutschland }\end{array}$ & Rollerpumpe & $\begin{array}{l}\text { Add-on zur CVVH, } \\
\text { bis } 400 \mathrm{ml} / \mathrm{min}\end{array}$ & Blutfluss, Drücke \\
\hline
\end{tabular}

ILA „interventional lung assist", PALP "pump assisted lung protection", $S_{V} O_{2}$ venöse Sauerstoffsättigung, $\mathrm{Hgb}$ Hämoglobin, $\mathrm{CVVH}$ kontinuierliche venovenöse Hämofiltration

Kursiv Derzeit nicht verfügbar, (DeCAP Smart, PALP) oder vor Markteinführung (Prismaflex-ECCO2-R)

um diverse Möglichkeiten erweitert worden. Die arteriovenöse pumpenlos perfundierte ILA war das erste verfügbare Verfahren im niedrigen bis mittlerem Blutflussbereich. Neuere Entwicklungen mit dem Ziel der $\mathrm{CO}_{2-}$
Elimination $\left(\mathrm{ECCO}_{2}-\mathrm{R}\right)$ waren und sind das DeCAP-Smart ${ }^{\circledR}$-System, das im Wesentlichen dem Prinzip einer kontinuierlichen rollerpumpengetriebenen Hämofiltration entspricht, in deren Kreislauf anstelle des Hämofilters eine
Gasaustauschmembran eingeschaltet ist, wie auch das Hemolung-RAS ${ }^{\circledR}$-System (A-Lung Technologies, Pittsburgh, USA) mit einer speziell für niedrige Blutflüsse optimierten Zentrifugalpumpe und Gasaustauschmembran. Beide Systeme arbeiten im Niedrigflussbereich („low flow“, 300-500 $\mathrm{ml} / \mathrm{min}$ ), dementsprechend werden relativ kleinlumige Doppellumenkanülen bis maximal $15 \mathrm{Fr}$ verwendet. Zwischen ECMO und $\mathrm{CO}_{2}$ Eliminationsverfahren stehen Systeme, die prinzipiell das gesamte Spektrum von niedrigen bis zu maximal möglichen Blutflüssen je nach Auswahl der Kanülen abdecken können und für die auch eine Auswahl an Schlauchsets inklusive Pumpenkopf und Membran für die jeweilige Indikation erhältlich ist. Ein Beispiel hierfür ist die iLA activve ${ }^{\circledR}$ (Novalung, Heilbronn, Deutschland), die mittels ein und derselben Konsole je nach verwendeten Komponenten das gesamte Spektrum abdecken kann. Zunehmend richtet sich der Fokus auch auf den sog. Mid-flow-Bereich zwischen etwa 1000 und $2000 \mathrm{ml} / \mathrm{min}$ Blutfluss, der durch eine relativ gering-invasive Konfiguration erreicht werden kann und zur effizienten $\mathrm{CO}_{2}$-Elimination, aber auch zu einer klinisch relevanten, wenn auch nicht vollständigen Oxygenierung führt [16]. Der Vorteil eines Niedrigflusssystems könnte im kleineren extrakorporalen Blutvolumen liegen sowie in der geringeren Invasivität durch kleinlumigere Kanülen respektive der Möglichkeit, mit einem einzigen venösen Gefäßzugang mittels Doppellumenkanüle auszukommen. Ob sich diese Strategie in einer besseren Verträglichkeit und einer geringeren Komplikationsrate niederschlägt, ist allerdings wissenschaftlich bislang nicht belegt.

\section{Kanülierung}

Die rezente Entwicklung der Kanülentechnik hat v. a. eine Reihe von Doppellumenkanülen hervorgebracht. Die Avalon-Elite ${ }^{\circledR}$-Kanülenserie (Maquet, Rastatt, Deutschland) reicht von kleinen, primär für die Pädiatrie gedachten Kanülen bis zu 31-Fr-Doppellumenkanülen für vollen ECMO-Support. Nachteile dieser Kanülen sind die aus technischen 
Gründen fehlende Heparinbeschichtung sowie die oft problematische perkutane Positionierung über die Vena jugularis interna dextra durch den rechten Vorhof in die Vena cava inferior, da die Kanüle sowohl über Ansaugöffnungen in der Vena cava superior als auch der Vena cava inferior drainiert. Fehllagen im rechten Ventrikel inklusive Ventrikelrupturen wurden beschrieben [18]. Auch die exakte Positionierung des Reperfusionsports in Richtung Trikuspidalklappe kann schwierig sein. Entsprechende Erfahrung sowie kompetente Beurteilbarkeit mittels Ultraschall sind eine Voraussetzung für die Verwendung dieser Technologie. Die Beschränkung auf einen singulären Venenzugang der oberen Körperhälfte erleichtert bei vielen Patienten allerdings die Lagerung bis hin zur vollen Mobilisierung beträchtlich.

Für niedrige und mittlere Blutflüsse wurden spezielle Kanülen entwickelt, die eine möglichst geringe Invasivität gewährleisten sollen. Für die arteriovenöse ILA werden optimierte heparinbeschichtete Kanülen mit Spezialkonnektor verwendet, wobei die Wahl des Kanülendurchmessers vom in der Ultraschalluntersuchung auszumessenden Gefäßdurchmesser abhängig ist (maximal 2 Drittel des arteriellen Gefäßquerschnitts). Für gepumpte venovenöse Verfahren mit dem vorrangigen Ziel einer $\mathrm{CO}_{2}$-Elimination stehen Doppellumenkanülen (Novaport Twin ${ }^{\circledR}$, Novalung) von 18, 22 und 24 Fr zur Verfügung, die Blutflüsse von etwa maximal $11 / \mathrm{min}$ (18 Fr) über $2 \mathrm{l} / \mathrm{min}(22 \mathrm{Fr}$ ) bis $\mathrm{zu}$ 2,5 1/min mittels 24-Fr-Kanüle ermöglichen. Für das Hemolung ${ }^{\circledR}$-System wird eine spezielle 15,5-Fr-Doppellumenkanüle für Blutflüsse zwischen 350 und $550 \mathrm{ml} / \mathrm{min}$ angeboten.

Die beschriebenen technischen Entwicklungen haben das Portfolio des extrakorporalen Gasaustauschs demnach beträchtlich erweitert, was den Klinikern wiederum vor das Problem stellt, sich im Vorhinein genau zu überlegen, welches Ziel beim jeweiligen Patienten erreicht werden soll. Es gilt die Regel: „so wenig invasiv wie möglich, jedoch so viel wie nötig.“ Klinisches Ziel sollte es sein, die Beatmungsinvasivität zu reduzieren oder überhaupt ohne Beatmung auszukom-

Med Klin Intensivmed Notfmed 2018 $113: 401-408$

https://doi.org/10.1007/s00063-016-0182-8

(c) Der/die Autor(en) 2016

\section{T. Staudinger}

\section{Extrakorporale Lungenersatzverfahren - was gibt es Neues, was kommt?}

\section{Zusammenfassung}

Technische Entwicklungen und die Erfahrungen aus der Influenzapandemie im Jahr 2009 haben die Anwendung von extrakorporalen Gasaustauschverfahren häufiger und sicherer werden lassen. Die Indikationsgebiete werden und wurden zunehmend erweitert. Neu auf den Markt gekommene Systeme haben die klassischen „High-flow-ECMO-Gasaustauschverfahren" - venovenöse und venoarterielle extrakorporale Membranoxygenation (ECMO) - um Systeme erweitert, die v. a. eine $\mathrm{CO}_{2}{ }^{-}$ Elimination zum Ziel haben, obwohl für viele propagierte Anwendungsgebiete profunde wissenschaftliche Evidenz fehlt.
Die Forschung erhellt dennoch zunehmend die pathophysiologischen Zusammenhänge und Interaktionen zwischen Organismus und extrakorporalem Verfahren. Neue Indikationen, wie Intubationsvermeidung und Reduktion der Beatmungsinvasivität, werden evaluiert. Neue Daten wie auch weitere technische Fortschritte werden neue und spannende Perspektiven schaffen.

\section{Schlüsselwörter}

$\mathrm{ECMO} \cdot \mathrm{ECCO}_{2}-\mathrm{R} \cdot$ Gasaustausch · Lungenversagen $\cdot$ Beatmung

\section{Extracorporeal lung support—news and future developments}

\section{Abstract}

Technical developments as well as the experiences during the 2009 influenza pandemia have led to an increased and safer use of extracorporeal gas exchange. Indications are expanding as new systems with the main goal of $\mathrm{CO}_{2}$ elimination have entered the market, thus, broadening the range of systems in addition to classic "high flow" extracorporeal membrane oxygenation (ECMO), although evidence for many suggested indications is sparse or lacking. However, recent research has shed light into the pathophysiology and interaction between the organism and the extracorporeal systems. Upcoming indications like avoiding intubation and mechanical ventilation or reducing invasiveness of ventilation are being evaluated. Novel data and technical advances will keep perspectives of extracorporeal gas exchange dynamic and exciting.

\section{Keywords}

$\mathrm{ECMO} \cdot \mathrm{ECCO}_{2}-\mathrm{R} \cdot \mathrm{Gas}$ exchange $\cdot$ Lung failure . Mechanical ventilation men. Mit einem System bzw. einer Konfiguration (= Kanülen, Schlauchsystem und Gasaustauschmembran), die Blutflüsse lediglich im Niedrigflussbereich ermöglicht, stößt man bei Patienten mit zusätzlichem Oxygenierungsproblem rasch an die Grenzen. Umgekehrt kann man mit einer klassischen ECMO durchaus über das Ziel hinausschießen und sich durch die großlumigen Kanülen, den möglicherweise höheren Bedarf an Antikoagulation und das hohe extrakorporal zirkulierende Volumen ein Mehr an Komplikationen einhandeln. Vorausschauendes Denken und Erfahrung sind demnach gefragt und im Zweifelsfall auch die Bereitschaft, die Konfiguration $\mathrm{zu}$ verändern und der Situation des $\mathrm{Pa}$ tienten anzupassen. Es sollte vermieden werden, ein invasives und potenziell mit schwerwiegenden Komplikation vergesellschaftetes Verfahren anzuwenden, ohne damit einen Vorteil für den $\mathrm{Pa}$ tienten $\mathrm{zu}$ erreichen. Als zusätzliches Problem erweist es sich nach wie vor, dass die Datenlage für viele der neuen Verfahren äußerst spärlich ist und man sich in vielen Indikationen nach den Kriterien einer evidenzbasierten Medizin auf dünnem Eis bewegt.

\section{Indikationen und neue Indikationen}

\section{ARDS}

Die klassische Indikation für die venovenöse ECMO ist nach wie vor das 
schwere ARDS mit lebensbedrohlicher Hypoxie. Die Frage, wann eine Hypoxie als lebensbedrohlich gewertet werden muss, ist schwer durch absolute Zahlen auszudrücken. Die Empfehlungen der Extracorporeal Life Support Organization (ELSO) können immerhin als Orientierungshilfe betrachtet werden (ECMO-Indikation bei ARDS mit $\mathrm{P}_{\mathrm{a}} \mathrm{O}_{2}$ $\mathrm{F}_{\mathrm{I}} \mathrm{O}_{2}$-Ratio $<100$ bei $\mathrm{F}_{\mathrm{I}} \mathrm{O}_{2}>0,90$ und einem „lung injury score“ von 2-3). Diese Empfehlungen spiegeln allerdings weder Geschichte und Zustand des Patienten, Beatmungseinstellungen und -invasivität, $\mathrm{CO}_{2}$-Austausch und Ausschöpfung anderer supportiver Maßnahmen, wie z.B. Bauchlagerung, inhalative Vasodilatatoren oder Recruitmentverfahren, wider. Die teils hohen Überlebensraten von ECMO-Patienten mit schwerstem Lungenversagen im Rahmen der Influenzapandemie des Jahres 2009 hat das Verfahren von der Ultima-RatioTherapie zur validen Alternative werden lassen [11]. Gleichzeitig haben die Ergebnisse der britischen "CESAR-Studie“ einen Überlebensvorteil durch Anwendung von ECMO bei ARDS suggeriert. Letztlich konnte diese viel kritisierte Studie einerseits eine hohe Überlebensrate von ARDS-Patienten mit ECMO zeigen, andererseits, dass ARDS-Therapie an einem spezialisierten Zentrum mit ECMO-Möglichkeit Überlebensvorteile bringen könnte [24]. Die Ausweitung der Indikation für ECMO bei ARDS weg von der reinen schweren Hypoxie hin zur Reduktion der Beatmungsinvasivität wäre eine logische Konsequenz. Randomisierte Daten dazu fehlen bislang. Eine laufende französische Multizenterstudie könnte diese Frage beantworten („EOLIA trial, clinical trials no. NCT01470703“). Die Daten aus dem Bereich der reinen $\mathrm{CO}_{2}$-Elimination, mit dem Ziel, die Beatmungsinvasivität zu reduzieren, zeigen zwar Hinweise auf eine Reduktion des beatmungsassoziierten Lungenschadens (VALI: [30]), jedoch konnte in einer prospektiven randomisierten Studie bei Patienten mit ARDS kein Überlebensvorteil zugunsten einer sog. ultraprotektiven Beatmung plus $\mathrm{ECCO}_{2}-\mathrm{R}$ im Vergleich zur konventionellen protektiven Beatmungsstrategie gezeigt werden. Die Studie war allerdings vorzeitig abgebrochen worden und zeigte immerhin eine signifikant verkürzte Beatmungsdauer in der Subgruppe mit schwererem ARDS [3]. Ein hoch-invasiver Beatmungsaufwand kann demnach nicht als evidenzgesicherte Indikation zur extrakorporalen $\mathrm{CO}_{2}$-Elimination gelten. Patienten mit Lungenversagen, die aufgrund einer respiratorischen Acidose nicht im Sinne der ARDS-Leitlinien protektiv zu beatmen sind, qualifizieren möglicherweise dennoch für $\mathrm{ECCO}_{2}-\mathrm{R}$. Die Beatmungsinvasivität lässt sich bei Patienten mit ARDS unter $\mathrm{ECCO}_{2}-\mathrm{R}$ rasch und effektiv reduzieren [34].

\section{Hyperkapnisches Lungenversagen}

Extrakorporale $\mathrm{CO}_{2}$-Elimination bei ausgeprägter respiratorischer Acidose ist im Sinne einer Normalisierung des $\mathrm{pH}$-Werts und einer Reduktion des Beatmungsaufwands hocheffektiv [2, 17, 29]. Die Methode wurde an obstruktiven Patienten, meist mit akut exazerbierter COPD (AECOPD), seltener im Status asthmaticus oder an Patienten mit terminalem restriktivem Lungenversagen in der Bridging-Situation zur Lungentransplantation erfolgreich angewendet. Neben der Beherrschung der Akutsituation bei akut hyperkapnischen Zuständen sind die Reduktion der Beatmungsinvasivität, das frühe Ermöglichen von Spontanatmung, die erleichterte Beatmungsentwöhnung und schließlich das Vermeiden von invasiver Beatmung an sich die möglichen Ziele einer $\mathrm{ECCO}_{2}-\mathrm{R}$ bei diesen Patienten.

Akut hyperkapnische Krisen sind durch $\mathrm{ECCO}_{2}-\mathrm{R}$ gut $\mathrm{zu}$ beherrschen. Eine Normalisierung des $\mathrm{pH}$-Werts kann in der Regel rasch erreicht werden. Die Beatmungsinvasivität lässt sich auch bei Patienten mit partieller respiratorischer Insuffizienz effektiv reduzieren [17]. $\mathrm{ECCO}_{2}-\mathrm{R}$ ist möglicherweise in der Lage, bei einem Teil der Patienten mit AECOPD eine Intubation zu verhindern $[6,22]$. Der Nachweis einer dadurch erreichten Verbesserung der Prognose wurde bis dato allerdings nicht erbracht. Ausgewählte Patienten mit terminalem restriktivem Lungenversagen oder chronischem Transplantatversagen (Bronchiolitis-obliterans-
Syndrom, BOS) können unter $\mathrm{ECCO}_{2}-\mathrm{R}$ erfolgreich und $\mathrm{zu}$ einem guten Teil spontan atmend zur Lungentransplantation oder Retransplantation geführt werden („bridging“; [13, 26]).

\section{Intubationsvermeidung}

Extrakorporaler Gasaustausch als Ersatz für die maschinelle Beatmung? Mit Bildern und Videos von an ECMO- oder $\mathrm{ECCO}_{2}$-R-Verfahren gehenden, spontan atmenden Patienten beeindrucken sowohl Intensivmediziner als auch Firmen ihr Publikum (• Abb. 2). In der Tat sind die modernen Systeme simpel und flexibel genug geworden, dass dies bei ausgewählten Patienten möglich wird. Erste Publikationen lassen potenzielle Indikationen erahnen [1]. ECMO und $\mathrm{ECCO}_{2}-\mathrm{R}$ sind mittlerweile als valide Verfahren zu betrachten, um Patienten mit terminalem Lungenversagen spontan atmend, mobilisierbar und mobil zur Lungentransplantation zu bringen. Im Vergleich zu historischen Kontrollen scheint in dieser speziellen Patientengruppe sich auch ein Outcomevorteil abzuzeichnen $[8,15,26]$.

Wie bereits erwähnt ist, kann $\mathrm{ECCO}_{2}-\mathrm{R}$ auch eine therapeutische Möglichkeit sein, um bei akut hyperkapnischen Krisen mit Versagen der nichtinvasiven Beatmung, wie der AECOPD, die Intubation vermeiden $\mathrm{zu}$ können. Hier steht der Nachweis einer verbesserten Prognose aus. Führt man sich das deutlich schlechtere Überleben der sekundär (also nach gescheiterter NIV) intubierten Patienten vor Augen [7], sollte im Umkehrschluss die Vermeidung einer Intubation $\mathrm{zu}$ besseren Ergebnissen führen. Zur „Wach-ECMO“ beim ARDS sind lediglich Einzelfälle oder kleine Serien in der Literatur zu finden. In einer Serie von 6, teilweise immunkompromittierten Patienten konnte die Intubation in der Hälfte der Fälle vermieden werden. Alle $3 \mathrm{~Pa}$ tienten überlebten, während von den 3 intubierten Patienten 2 verstarben [19]. In einem Fall kam es zur Selbstentfernung einer ECMO-Kanüle durch einen der Patienten. Die Anwendung von ECMO am wachen Patienten ist demnach zweifellos als experimentelles 
Tab. 2 Gerinnungsstörungen unter ECLS

\begin{tabular}{|c|c|c|}
\hline Gerinnungsstörung & Klinisches Bild & Diagnostik \\
\hline Thrombosierung Oxygenator & Hypoxie, evtl. Hämolyse & $\begin{array}{l}\mathrm{TMP} \uparrow, \mathrm{POxy}-\mathrm{pO}_{2} \downarrow \text {, D-Dimer } \uparrow \text {, } \\
\mathrm{PLT} \downarrow \text {, (Hämolyse?) }\end{array}$ \\
\hline Thrombosierung Pumpenkopf & Hämolyse & $\begin{array}{l}\text { Geräusche im Pumpenkopf, } \\
\text { Hämolyse }\end{array}$ \\
\hline $\begin{array}{l}\text { Überantikoagulation (Hepa- } \\
\text { rin) }\end{array}$ & Blutung & $\begin{array}{l}\text { aPTT }>80 \mathrm{~s}, \mathrm{TZ} \uparrow \uparrow, \text { Anti-Xa } \\
>0,50-0,60\end{array}$ \\
\hline Venöse Thrombosen & Evtl. Pulmonalembolie & D-Dimer $\uparrow$, Ultraschall \\
\hline $\begin{array}{l}\text { Thrombozytopenie ("con- } \\
\text { sumption“) }\end{array}$ & Evtl. Blutung & PLT $\downarrow$ \\
\hline DIC & "Clotting", Blutungsneigung & $\begin{array}{l}\text { Fbg } \downarrow, \text { PTZ und aPTT } \uparrow, \text { PLT } \downarrow \text {, } \\
\text { D-Dimer } \uparrow, F_{1+2} \uparrow\end{array}$ \\
\hline Hyperfibrinolyse & Blutung & D-Dimer $\uparrow$, Fbg $\downarrow$, TEM \\
\hline $\begin{array}{l}\text { Erworbenes von-Willebrand- } \\
\text { Syndrom }\end{array}$ & Blutung & vWF:RCo/vWF: Ag-Ratio < 0,6 \\
\hline \multicolumn{3}{|c|}{ 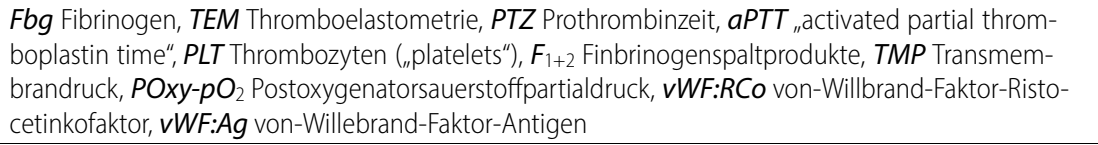 } \\
\hline
\end{tabular}

Verfahren zu betrachten und sollte sehr erfahrenen Zentren bei ausgewählten Patienten vorbehalten werden. Als erster Schritt kann jedenfalls eine Reduktion der Analgosedierung bei stabil laufendem extrakorporalem Verfahren mit dem Ziel der (teilweisen) Spontanatmung, der Kommunikationsfähigkeit und - in letzter Konsequenz - der assistiven bzw. aktiven Mobilisierung angedacht werden.

\section{Neue Indikationen}

Der Schritt weg vom Tubus hin zur erhaltenen Spontanatmung unter extrakorporalem Gasaustausch ist demnach bei weitem nicht vollzogen und ist aus heutiger Sicht lediglich für ausgewählte Patienten geeignet. Die nach wie vor beträchtliche Rate an teilweise auch schweren Komplikationen durch extrakorporale pumpengetriebene Verfahren in der Langzeitanwendung ist einer der Gründe hierfür. Derzeit mehren sich immerhin die Daten zur Anwendung bei AECOPD und in der Überbrückung zur Lungentransplantation.

Die schlechte Prognose immunsupprimierter Patienten mit Lungenversagen, insbesondere von hämatologischen und onkologischen Patienten, hat in ersten Ansätzen zur Überlegung geführt, ob eine Vermeidung der Intubation mithilfe von extrakorporalem Gasaustausch zu einer höheren Erfolgsrate führen könnte. In einer Serie selektierter - allerdings durchwegs beatmeter - hämatologischer Patienten konnte unsere Gruppe eine überraschend hohe Überlebensrate zeigen [33], für knochenmarkstransplantierte Patienten sehen die ersten Datenanalysen wesentlich weniger positiv aus [32]. Überraschenderweise führte die oft bestehende ausgeprägte Thrombozytopenie bei diesen Patienten nicht $\mathrm{zu}$ fatalen Blutungskomplikationen und kann demnach nicht mehr als absolute Kontraindikation für ECLS betrachtet werden.

\section{Patientenmanagement}

\section{Interaktionen}

Extrakorporaler Gasaustausch interagiert mit der Physiologie des Patienten in vielfältiger Weise. Das Bewusstsein, dass es sich dabei nicht um ein rein technisches Verfahren handelt, das unabhängig von Körperfunktionen das Blut oxygeniert bzw. decarboxyliert, hat sich in den letzten Jahren zunehmend entwickelt. Angefangen von den eingangs erwähnten technischen Entwicklungen mit deutlicher Verringerung von Hämolyse, Gerinnungs- und Entzündungsaktivierung mehren sich Untersuchungen zu physiologischen Effekten durch die Systeme. Es konnte gezeigt werden, dass komplexe Veränderungen der Blutgerinnung zu beachten sind und einen wesentlich höheren Aufwand an Diagnostik als Gerinnungsglobaltests erfordern (• Tab. 2). Erst seit dem Jahr 2014 liegen erste Daten vor, die für eine protektive „Open-lung-Beatmungsstrategie“ für ARDS-Patienten unter ECMO sprechen, ein Umstand der lange umstritten war und teilweise einer Strategie der minimalen Ventilation bis hin zur Totalatelektase Vorschub geleistet hat [27].

Die Interaktion v. a. der High-flowSysteme mit der Physiologie des Kreislaufsystems stellt ein wichtiges Kriterium für die Effizienz einer ECMO dar. Für VA-Systeme ist dies seit langem bekannt. Als Beispiel sei das Problem der "differenziellen Hypoxie“ bei femorofemoraler Konfiguration genannt (= Hypoxie der oberen Körperhälfte bei Lungenversagen und guter linksventrikulärer Funktion und dadurch bedingter Versorgung des Aortenbogens mit Blut aus dem linken Ventrikel anstatt mit Blut aus dem extrakorporalen System), das zu alternativen Kanülierungsstrategien geführt hat $[5,9]$. Erst im Jahr 2013 hat eine französische Gruppe die Zusammenhänge zwischen eingestellten Blut- und Gasflussparametern am Gerät und physiologischen Veränderungen systematisch untersucht [28]. Nicht überraschend konnte gezeigt werden, dass eine Erhöhung des Gasflusses $\mathrm{zu}$ einer gesteigerten $\mathrm{CO}_{2}$ Elimination führt, während eine Reduktion der $\mathrm{F}_{\mathrm{I}} \mathrm{O}_{2}$ im Gasfluss eine rasch schlechtere Oxygenierung bei gleichzeitig unveränderter $\mathrm{CO}_{2}$-Elimination beim Patienten bewirkt. Eine wesentliche, wenn auch nicht neue Erkenntnis ist der Zusammenhang zwischen ECMOBlutfluss und Herzzeitvolumen (HZV): Erst ab einem Blutfluss $\geq 60 \%$ des HZV konnte eine deutliche Reduktion des Beatmungsaufwands in Richtung protektiv, respektive ultraprotektiv erreicht werden. Die Höhe des HZV entspricht der Menge des venösen Rückstrombluts, dem unter venovenöser ECMO das extrakorporal oxygenierte Blut beigemischt wird. Je höher also der Anteil des ECMO-Blutflusses am HZV ist, desto besser oxygeniertes Blut gelang über 


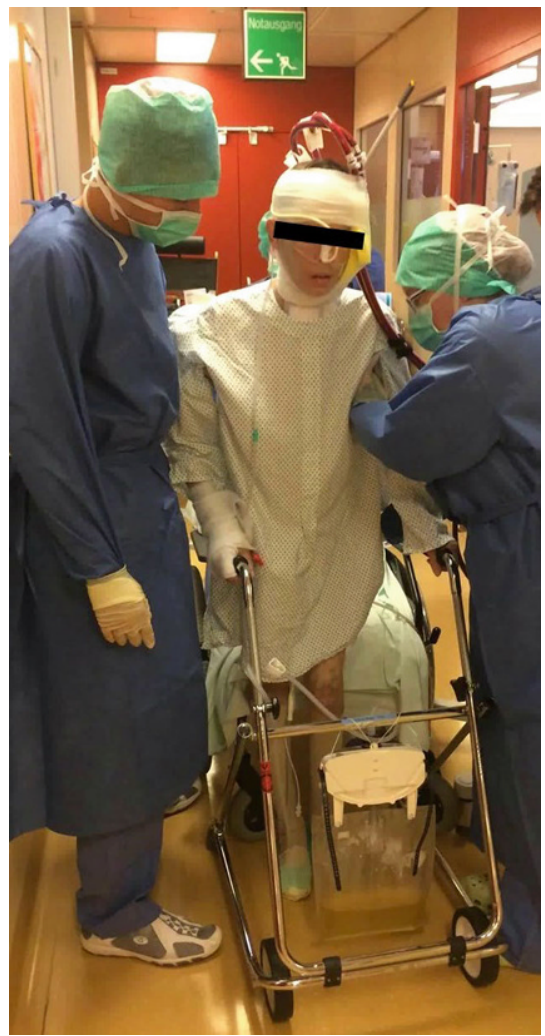

Abb. $2 \Delta$ Patientin mit Lungenfibrose nach ARDS mit jugulärer Doppellumenkanüle und iLA activve ${ }^{\circledR}$ während der Mobilisierung. Erfolgreiches Weaning nach 7-wöchiger Beatmung am ECLS und "bridging" zur Lungentransplantation

die Lungenstrombahn in das arterielle System. Moderne ECMO-Systeme bieten neben Druckmonitoring auch die kontinuierliche Messung der venösen Sauerstoffsättigung (= vor Durchströmen des Oxygenators), der als Maß für ein adäquates Sauerstoffangebot zur Steuerung des ECMO-Blutflusses besondere Bedeutung zukommt. Neben dem HZV und der Sauerstoffsättigung spielt auch die Menge des Hämoglobins für den Sauerstofftransport im Organismus eine wesentliche Rolle und sollte bei Patienten an der VV-ECMO entsprechend überwacht und beachtet werden. Aus dem Gesagten ergibt sich auch die Notwendigkeit eines HZV-Monitorings beim Patienten. Neben der klassischen Messung mittels Pulmonalarterienkatheter oder PiCCO ${ }^{\mathrm{max}}$ (PULSION Medical Systems SE, Feldkirchen, Deutschland) steht heute eine Reihe weniger invasiver Verfahren zur Verfügung, die auf Ultraschalltechnologie (Echokardiographie, transösophageale Dopplersonographie) oder Pulskurvenanalyse beruhen.

\section{Lagerung, Mobilisierung}

Extrakorporale Gasaustauschverfahren sind überbrückende Verfahren, die den zugrunde liegenden krankhaften Zustand nicht verbessern. Ihr Therapieziel ist ein rein symptomatisches. Dementsprechend gilt die Auffassung, aufgrund der unter ECLS erreichten respiratorischen Stabilität eine möglichst passive Behandlungsstrategie zu wählen, mittlerweile als obsolet. Unter jedweder Form der ECLS sind folgende adjuvante therapeutische Strategien grundsätzlich möglich:

- Lagerungstherapie inkl. Bauchlagerung und ggf. auch kontinuierlicher lateraler Rotationstherapie,

- diagnostische Verfahren inkl. Bildgebung außerhalb der Intensivstation (z. B. CT),

- Reduktion der Analgosedierung mit Einsetzen zumindest teilweiser Spontanatmung und Kontaktierbarkeit,

- Weaning vom Respirator bis hin zur Extubation,

- Frühmobilisation bis hin zur aktiven Vertikalisierung und zum Gehen.

Die Umsetzbarkeit solcher Maßnahmen ist in hohem Maß von Erfahrung, personeller Ausstattung und der spezifischen Situation der Patienten abhängig. Schrittweise vorsichtige Annäherung in enger interdisziplinärer Zusammenarbeit bis zu der im Moment erreichbaren Grenze muss die Maxime für die Therapiestrategie bleiben. Dennoch muss heutzutage ein aufgrund des bloßen Vorhandenseins eines extrakorporalen Gasaustauschverfahrens tief analgosedierter flach auf dem Rücken gelagerter Patient ohne Spontanatmungsanteile als gut zu begründender Ausnahmefall betrachtet werden.

\section{Zukünftige Entwicklungen}

Im kommenden Jahr ist mit der Markteinführung von Hämofiltrationssystemen mit der Möglichkeit einer zusätzlichen $\mathrm{CO}_{2}$-Elimination zu rechnen. Auf dem Konzept des DeCAP-Smart ${ }^{\circledR}$-Systems beruhend soll bei Blutflüssen bis maxi$\mathrm{mal} 400 \mathrm{ml} / \mathrm{min}$ das $\mathrm{CO}_{2}$ um etwa $20 \%$ reduziert werden. Im Gegensatz zum DeCAP-Smart ${ }^{\circledR}$-System ist eine Kombination mit Hämofiltration möglich. Geringe Invasivität und die Nutzung ein und desselben Geräts für beide Verfahren sind die von der Industrie genannten Vorteile. Zusätzlich wäre möglicherweise die mittlerweile zum Standard gewordene regionale Antikoagulation mit Zitrat bei diesen Verfahren möglich. Ziel der moderaten $\mathrm{ECCO}_{2}-\mathrm{R}$, die auf diese Weise möglich ist, kann letztlich v. a. die Reduktion der Beatmungsinvasivität sein.

Bei den Herstellern von ECLS-Systemen wird die Entwicklung in Richtung Kompaktheit und einfache Bedienung sowie eine Erweiterung des systemimmanenten Monitorings gehen. Schon heute ist eine Überwachung der Sog- und Transmembrandrücke sowie von wesentlichen physiologischen Messwerten, wie von Hämoglobin und venöser Sauerstoffsättigung, zu fordern (• Abb. 2). Fortschritte in der Beschichtungstechnologie von Schlauchsystemen und Kanülen zur weiteren Verbesserung der Biokompatibilität sind ebenfalls zu erwarten. Experimentelle Ansätze zur Vermeidung von Gerinnungsaktivierung an den Fremdoberflächen haben eine Verringerung oder sogar den Wegfall der Antikoagulation zum Ziel.

Neue Entwicklungen in der Kanülentechnik haben das Ziel einer geringeren Komplikationsrate bei gleicher oder gesteigerter Effizienz gemeinsam. Eine biokompatible Beschichtung auf Heparinbasis soll die Performance der Avalon-Elite ${ }^{\circledR}$-Doppellumenkanüle in naher Zukunft verbessern. Stentbasierte venöse Drainagesysteme sollen die Problematik des „Ansaugens" hintanhalten und höhere Flussraten ermöglichen [25]. Ein interessantes Konzept birgt auch eine neu entwickelte Doppellumenkanüle, die perkutan über die Vena cava superior, wo die Blutdrainage erfolgt, über den rechten Vorhof und Ventrikel in die Pulmonalarterie (Reperfusionsauslass) positioniert wird. Der rechte Ventrikel wird damit umgangen und volumenentlastet, sodass ein quasi venovenöses Verfahren auch bei 
Patienten mit schlechter Rechtsventrikelperformance möglich erscheint [31].

Rezente Daten werden Einfluss auf die Antikoagulationsstrategie nehmen: Thrombozytenaggregationshemmer scheinen einen positiven Effekt auf die Clotting-Ereignisse im extrakorporalen Kreislauf zu haben und könnten zukünftig als Kombinationspartner von Heparin $\mathrm{zu}$ einer effektiveren Gerinnungshemmung bei geringerem Heparinbedarf führen [4]. Als Gerinnungsmonitoring scheint der Anti-Xa-Spiegel eine bessere Korrelation mit der Heparindosis zu bieten als z.B. ACT oder aPTT [20]. Alternative Substanzen, wie Argatroban oder Bivalirudin, stehen zur Verfügung. Größer angelegte Studien werden allerdings nötig sein, um den derzeitigen Standard zu ändern.

\section{Resümee und Ausblick}

Technische Neuentwicklungen haben in den letzten Jahren Eingang in die klinische Praxis gefunden. Extrakorporale Gasaustauschverfahren werden längst auch außerhalb großer ECMO-Zentren in breitem Umfang angewendet. Erst langsam nimmt die wissenschaftliche Evidenz für die Verwendung dieser Systeme zu. Erfahrung und die Kenntnis der Systeme, ihrer Effekte und der komplexen Interaktionen mit der Physiologie sind die Voraussetzung für eine sichere und erfolgreiche Anwendung. Eine entsprechende Ausstattung, das Vorhandensein von Spezialisten unterschiedlicher Disziplinen sowie eine Mindestanzahl von Anwendungen pro Jahr sind zu fordern. Die ELSO empfiehlt diesbezüglich mindestens 6 Patienten [12], während ein internationales Expertengremium die Behandlung von mehr als 20 Patienten pro Jahr fordert [10]. Tatsächlich konnte zumindest im pädiatrischen $\mathrm{Be}$ reich eine positive Assoziation zwischen Anwendungshäufigkeit und Prognose gezeigt werden [14, 21]. Experimentelle Indikationen sollten erfahrenen Zentren unter Studienbedingungen vorbehalten bleiben. Die nächsten Jahre werden eine Fülle von Daten erbringen, die gemeinsam mit weiteren technischen Fortschritten - eine differenzierte und fundierte Anwendung der Verfahren ermöglichen wird. Mit Spannung dürfen jedenfalls neue wissenschaftlich fundierte Erkenntnisse und die Entwicklung der nächsten Jahre erwartet werden.

\section{Fazit für die Praxis}

\section{- Technische Entwicklungen der letz- ten Jahre haben die Anwendung ex- trakorporaler Gasaustauschsysteme einfacher und komplikationsärmer gemacht.}

- Eine Vielzahl individuell zu adaptierender Systeme und Konfigurationen stehen zur Verfügung.

- Die steigende Zahl möglicher Indikationen führt bereits jetzt zu einer zunehmend breiten Anwendung der verfügbaren Systeme.

- Für viele Einsatzmöglichkeiten fehlen - trotz intensiver Forschungstätigkeit - valide klinische Daten, sodass diese als experimentell betrachtet werden müssen.

- Erfahrung und die Kenntnis der Systeme, ihrer Effekte und der komplexen Interaktionen mit der Physiologie sind die Voraussetzung für ihre sichere und erfolgreiche Anwendung.

- Eine entsprechende Ausstattung, das Vorhandensein von Spezialisten unterschiedlicher Disziplinen sowie eine Mindestanzahl von Anwendungen pro Jahr sind zu fordern.

\section{Korrespondenzadresse}

\section{Univ.-Prof. Dr. T. Staudinger}

Universitätsklinik für Innere Medizin I, Intensivstation 13.i2, Allgemeines Krankenhaus der Stadt Wien/Medizinische Universität Wien Währinger Gürtel 18-20, 1090 Wien, Österreich thomas.staudinger@meduniwien.ac.at

Open access funding provided by Medical University of Vienna

\section{Einhaltung ethischer Richtlinien}

Interessenkonflikt. T. Staudinger erhielt, außerhalb der vorgelegten Arbeit, Personalmittel von Novalung, Maquet und Zoll.

Dieser Beitrag beinhaltet keine von den Autoren durchgeführten Studien an Menschen oder Tieren.

Open Access. Dieser Artikel wird unter der Creative Commons Namensnennung 4.0 International Li- zenz (http://creativecommons.org/licenses/by/4.0/ deed.de) veröffentlicht, welche die Nutzung, Vervielfältigung, Bearbeitung, Verbreitung und Wiedergabe in jeglichem Medium und Format erlaubt, sofern Sie den/die ursprünglichen Autor(en) und die Quelle ordnungsgemäßnennen, einen Linkzur Creative Commons Lizenz beifügen und angeben, ob Änderungen vorgenommen wurden.

\section{Literatur}

1. Abrams D, Javidfar J, Farrand E et al (2014) Early mobilization of patients receiving extracorporeal membrane oxygenation: a retrospective cohort study. Crit Care 18:R38

2. Abrams D, Roncon-Albuquerque R Jr, Brodie D (2015) What's new in extracorporeal carbon dioxide removal for COPD? Intensive Care Med 41:906-908

3. Bein T, Weber-Carstens $S$, Goldmann A et al (2013) Lower tidal volume strategy $(\approx 3 \mathrm{ml} / \mathrm{kg}$ ) combined with extracorporeal $\mathrm{CO} 2$ removal versus 'conventional' protective ventilation $(6 \mathrm{ml} / \mathrm{kg})$ in severe ARDS: the prospective randomized Xtravent-study. Intensive Care Med 39:847-856

4. Bein T, Zimmermann M, Philipp A et al (2011) Addition of acetylsalicylic acid to heparin for anticoagulation management during pumpless extracorporeal lung assist. ASAIO J 57:164-168

5. Biscotti M, Barchetta M (2014) The "sport model": extracorporeal membrane oxygenation using the subclavian artery. Ann Thorac Surg 98:1487-1489

6. Burki NK, Mani RK, Herth FJ et al (2013) A novel extracorporeal $\mathrm{CO} 2$ removal system: results of a pilot study of hypercapnic respiratory failure in patients with COPD. Chest 143:678-686

7. Chandra D, Stamm JA, Taylor B et al (2012) Outcomes of noninvasive ventilation for acute exacerbations of chronic obstructive pulmonary disease in the United States, 1998-2008. Am J Respir Crit Care Med 185:152-159

8. Chiumello D, Coppola S, Froio S et al (2015) Extracorporeal life support as bridge to lung transplantation: a systematic review. Crit Care 19:19

9. Choi JH, Kim SW, Kim YU et al (2014) Application of veno-arterial-venous extracorporeal membrane oxygenation in differential hypoxia. Multidiscip Respir Med 9:55

10. Combes A, Brodie D, Bartlett R (2014) Position paper for the organization of extracorporeal membrane oxygenation programs for acute respiratory failure in adult patients. Am J Respir Crit Care Med 190:488-496

11. Davies A, ANZECMO Investigators (2009) Extracorporeal Membrane Oxygenation for 2009 Influenza A(H1N1) Acute Respiratory Distress Syndrome. JAMA 302:1888-1895

12. ELSO guidelines for ECMO centers. https://www. elso.org/Portals/0/IGD/Archive/FileManager/ faf3f6a3c7cusersshyerdocumentselsoguide linesecmocentersv1.8.pdf. Zugegriffen: 2. März 2016

13. Fischer S, Simon AR, Welte T et al (2006) Bridge to lung transplantation with the novel pumpless interventional lung assist device NovaLung. JThorac Cardiovasc Surg 131:719-723

14. Freeman $C L$, Bennett TD, Casper $T C$ et al (2014) Pediatric and neonatal extracorporeal membrane oxygenation: does center volume impact mortality? Crit Care Med 42:512-519

15. Fuehner T, Kuehn C, Hadem J et al (2012) Extracorporeal membrane oxygenation in awake 
patients as bridge to lung transplantation. Am J Respir Crit Care Med 185:763-768

16. Hermann A, Riss K, Schellongowski P et al (2015) A novel pump-driven veno-venous gas exchange system during extracorporeal $\mathrm{CO} 2-$ removal. Intensive Care Med 41:1773-1780

17. Hermann A, Staudinger T, Bojic A et al (2014) First experience with a new miniaturized pump-driven venovenous extracorporeal $\mathrm{CO} 2$ removal system (iLA Activve): a retrospective data analysis. ASAIO J 60:342-347

18. Hirose H, Yamane K, Marhefka G et al (2012) Right ventricular rupture and tamponade caused by malposition of the avalon cannula for venovenous extracorporeal membrane oxygenation. JCardiothorac Surg 7:36

19. Hoeper MM, Wiesner O, Hadem J et al (2013) Extracorporeal membrane oxygenation instead of invasive mechanical ventilation in patients with acute respiratory distress syndrome. Intensive Care Med 39:2056-2057

20. Irby K, Swearingen C, Byrnes J et al (2014) Unfractionated heparin activity measured by antifactor Xa levels is associated with the need for extracorporeal membrane oxygenation circuit/ membrane oxygenator change: a retrospective pediatric study. Pediatr Crit Care Med 15:175-182

21. Karamlou T, Vafaeezadeh M, Parrish AMet al (2013) Increased extracorporeal membrane oxygenation center case volume is associated with improved extracorporeal membrane oxygenation survival among pediatric patients. JThorac Cardiovasc Surg 145:470-475

22. Kluge S, Braune SA, Engel M et al (2012) Avoiding invasive mechanical ventilation by extracorporeal carbon dioxide removal in patients failing noninvasive ventilation. Intensive Care Med 38:1632-1639

23. Moen O, Høgåsen K, Fosse $E$ et al (1997) Attenuation of changes in leukocyte surface markers and complement activation with heparincoated cardiopulmonary bypass. Ann Thorac Surg 63:105-111

24. Peek GJ, Mugford M, Tiruvoipati R et al (2009) Efficacy and economic assessment of conventional ventilatory support versus extracorporeal membrane oxygenation for severe adult respiratory failure (CESAR): a multicentre randomised controlled trial. Lancet 374:1351-1363

25. Piñón M, Pradas G, Molina D et al (2015) Use of self-expanding venous cannula in tricuspid reoperation. Eur J Cardiothorac Surg 48:499-501

26. Schellongowski P, Riss K, Staudinger T et al (2014) Extracorporeal $\mathrm{CO} 2$ removal as bridge to lung transplantation in life-threatening hypercapnia. Transpl Int 28:297-304

27. Schmidt M, Pellegrino V, Combes A et al (2014) Mechanical ventilation during extracorporeal membrane oxygenation. Crit Care 18:203

28. Schmidt M, Tachon G, Devilliers Cetal (2013) Blood oxygenation and decarboxylation determinants during venovenous ECMO for respiratory failure in adults. Intensive Care Med 39:838-846

29. Sklar MC, Beloncle F, Katsios CM (2015) Extracorporeal carbon dioxide removal in patients with chronic obstructive pulmonary disease: a systematic review. Intensive Care Med 41:1752-1762

30. Terragni PP, Del Sorbo L, Mascia L et al (2009) Tidal volume lower than $6 \mathrm{ml} / \mathrm{kg}$ enhances lung protection: role of extracorporeal carbon dioxide removal. Anesthesiology 111:826-835

31. Wang D, Jones C, Ballard-Croft $C$ et al (2015) Development of a double-lumen cannula for a percutaneous RVAD. ASAIO J61:397-402
32. Wohlfarth P, Beutel G, Lebiedz P et al (2015) Veno-venöse ECMO bei Patienten mit ARDS nach allogener Stammzelltransplantation. Med Klin Intensivmed Notfmed 110:293-321 (AbstractP18)

33. Wohlfarth P, Ullrich R, Staudinger T et al (2014) Extracorporeal membrane oxygenation in adult patients with hematologic malignancies and severe acute respiratory failure. Crit Care 18:R20

34. Zimmermann M, Bein T, Arlt M et al (2009) Pumpless extracorporeal interventional lung assist in patients with acute respiratory distress syndrome: a prospective pilot study. Crit Care 13:R10
In eigener Sache

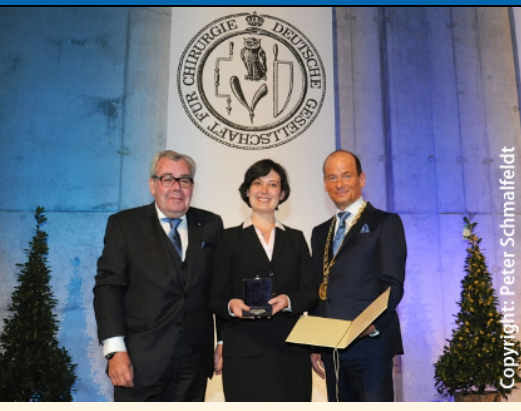

Redakteurin des Springer Medizin Verlags mit der Werner-Körte-Medaille in Silber 2018 ausgezeichnet

Die traditionsreiche Werner-Körte-Medaille in Silber wurde am 17.04.2018 in Berlin von der Deutschen Gesellschaft für Chirurgie (DGCH) vergeben. Der Namensgeber Professor Dr. Werner Körte (1853 - 1937) war praktizierender Chirurg in Berlin, erster Schriftführer der DGCH sowie deren Präsident und Ehrenvorsitzender. Im Rahmen der feierlichen Eröffnungsveranstaltung des 135 . Kongresses der Deutschen Gesellschaft für Chirurgie wurde die Auszeichnung an Christiane Jurek, Managing Editor, Redaktion Fachzeitschriften Medizin/Psychologie der Springer Medizin Verlag $\mathrm{GmbH}$, verliehen. Mit diesem Preis würdigte das Präsidium der DGCH ihre langjährigen Verdienste um die Förderung der wissenschaftlichen Chirurgie, für die sie sich persönlich mit ihrem Unternehmen in besonderer Weise engagiert hat. Herr Professor Dr. Jörg Fuchs, Präsident der DGCH und Herr Professor Dr. Dr. h. c. HansJoachim Meyer, Generalsekretär der DGCH, vergaben den Preis an diesem Abend. „Es ist mir eine große Ehre, über meine Tätigkeit bei der Zeitschrift Der Chirurg mit den Vertretern der DGCH vertrauensvoll zusammen arbeiten zu dürfen. Die Gesellschaft ist eine der ältesten medizinischwissenschaftlichen Fachgesellschaften, die sich seit jeher bemüht, die politischen, ökonomischen und sozialen Rahmenbedingungen in der Chirurgie zu verbessern. Der Springer Medizin Verlag versteht sich als starker Partner der DGCH und ist stolz auf die gemeinsame Zusammenarbeit", so Frau Jurek.

Springer Medizin, Heidelberg 